\title{
Successful Treatment of a Transsphincteric Perianal Fistula Using a Combination of the FiLAC and Lift Techniques
}

\section{Georgios K. Georgiou}

Iasi Private Medical Center, 75 Dodonis avenue, 45221 Ioannina, Greece
Abstract
Background: Perianal fistulas are difficult to treat. Several minimally invasive sphincter-sparing methods have been described with encouraging results.
Publication History:
Received: December 24, 2017
Accepted: February 26, 2018
Published: February 28, 2018
Methods: A 48-year-old male patient with a transsphincteric fistula was operated using both FiLAC and LIFT techniques.
Results: successful treatment was witnessed as fistula closure within 3 weeks and absence of recurrence at 7 months follow-up
Keywords:
Fistula, Transsphincteric, Laser, Diode

Conclusion: This is the first report of successful perianal fistula treatment using a combination of FiLAC and LIFT techniques

\section{Introduction}

Treatment of perianal fistulas has always been challenging, balancing between long-term effectiveness (with higher success rates when using more aggressive surgical techniques) and the risk of postoperative sphincter dysfunction [1]. Many new, minimal invasive techniques have appeared lately, such as eradication of the fistula using novel biomaterials, the Video-assisted anal fistula treatment (VAAFT) technique or the OTSC ${ }^{\text {TM }}$ Proctology system (Ovesco Endoscopy AG, Tuebingen, Germany) [2].

One of the most promising methods currently applied is the ligation of inter-sphincteric fistula tract (LIFT), which was first described by Rojanasakul in 2007 [3]. Curettage of the external opening up to the level of the external sphincter muscle completes the procedure in some cases, known as LIFT Plus [4]. On the other hand, the Fistula Laser Closing (FiLaC ${ }^{\mathrm{TM}}$ ) device (Biolitec, Germany) has shown good primary results in both cryptoglandular and Crohn's anal fistulas and even better if used for a second time $[5,6]$. However, some authors admit that the laser procedure alone may be insufficient in closing the internal fistula opening, which may be implicated in fistula recurrence [6].

It appears that we are still far from an ideal, universally accepted method for successful treatment of anal fistulas, since each individual technique has its pros and cons. In the case of a transsphincteric anal fistula that is presented here, a combination of the FiLAC and LIFT surgical techniques was preferred for optimal results.

\section{Case Presentation}

A 48-year-old male patient presented to my office suffering from an anal fistula during the past 8 months, after having been operated elsewhere for a perianal abscess. His medical history included rheumatoid arthritis under etanercept (subcutaneous injections once every week), leflunomide (20 mg per os once daily) and methylprednisolone (4 mg per os once daily), as well as arterial hypertension under valsartan $80 \mathrm{~g}$ once daily. Clinical examination revealed an external fistula opening at 8 o'clock at lithotomy position with purulent discharge. A Magnetic Resonance Imaging (MRI) was ordered, revealing an active transsphincteric fistula tract with an internal opening at 8 o'clock, while the intersphincteric space showed signs of ongoing sepsis. A seton placement was arranged under local anaesthesia and was left in place for the subsequent 3 months, in order to permit the sepsis to subside. A new MRI study was performed, showing elimination of the intersphincteric infection and absence of secondary fistulous tracts.

The patient was operated under spinal anaesthesia in the jack-knife position. Firstly the seton was removed and replaced by a metallic probe which was used as a guide in order to facilitate identification of the fistula tract. Typical LIFT procedure was performed by meticulous dissection through the intersphincteric plane, double suture ligation of the fistula tract using Vicryl No 2/0 and finally cutting of the fistula tract between the sutures. The intersphincteric plane was further explored for residual cryptoglandular infection foci and thoroughly cleansed. Adequacy of ligation at the side of the external sphincter was confirmed by non-passing of hydrogen peroxide solution which was instilled from the external fistula opening through an abbocath. The lateral portion of the fistulous tract (from the external sphincter towards the external opening at the ischiorectal fossa) was then obliterated using a diode laser emitting energy in a circumferential manner (radial optical fiber) with a wavelength of $1.470 \mathrm{~nm}$ (BiolItec). The generator was set at $13 \mathrm{~W}$ providing pulsed energy shots of $1 \mathrm{sec}$ with a $0.5 \mathrm{sec}$ "dead" interval, during which the fiber was pulled to the next position of energy delivery, moving from medial to lateral, as in standard FiLAC procedure. Total amount of energy delivered was 510 Joules for a fistula tract with a length of $3.5 \mathrm{~cm}$. the external fistula opening was left intact and the perianal area was covered with sterile gauzes.

Post-operatively, the patient was administered 500 mg of Cefuroxime per os twice daily and $500 \mathrm{mg}$ of Metronidazole trice daily for 5 days. Simple painkillers (paracetamol and ibuprofen) were prescribed and used by the patient on demand. The patient was discharged after an overnight stay and checked at week intervals for fistula healing or ongoing discharge. His course was uneventful and 3 weeks after the procedure the fistula had healed, as witnessed in clinical examination

*Corresponding Author: Dr. Georgios K. Georgiou, lasi Private Medical Center 75 Dodonis avenue, 45221 loannina, Greece, Tel: + 30 6942964111, Fax: + 30 2651074001; Email: georgmd7@gmail.com

Citation: Georgiou GK (2018) Successful Treatment of a Transsphincteric Perianal Fistula Using a Combination of the FiLAC and Lift Techniques. Int J Surg Surgical Porced 3: 133. https://doi.org/10.15344/2456-4443/2018/133

Copyright: @ 2018 Georgiou. This is an open-access article distributed under the terms of the Creative Commons Attribution License, which permits unrestricted use, distribution, and reproduction in any medium, provided the original author and source are credited. 
Citation: Georgiou GK (2018) Successful Treatment of a Transsphincteric Perianal Fistula Using a Combination of the FiLAC and Lift Techniques. Int J Surg Surgical Porced 3: 133. https://doi.org/10.15344/2456-4443/2018/133

Page 2 of 2

and transdermal ultrasonographic evaluation. Today, seven months after the operation, there are no clinical or radiological (new MRI) proofs of recurrence.

\section{Discussion}

FiLaC is a promising sphincter-saving procedure for anal fistula that seems to have a high success rate and low morbidity. It is not technically demanding and allows for quick recovery [7]. In the largest study of perianal fistulas treated with the FiLAC technique, Wilhelm et al., report primary healing rate of $64.1 \%$ ( 75 out of 117 patients over a 12-month period of follow-up), with no difference between fistulas of cryptoglandular origin and those related to Crohn's disease [6]. The secondary success rate (that is after a second application of the FiLAC technique) was reported to be $88 \%$. It is interesting to note that the above mentioned success rates refer to all types of fistula combined (according to Parks classification), thus making FiLAC a near-ideal treatment for both simple and complex anal fistulas [8].

However, in many cases the surgeons combine this technique with some other procedure such as suture-closure of the internal opening or creation of an endorectal advancement flap [9]. This is to ensure that septic material will not be allowed to pass alongside the fistula tract during its period of healing, thus raising the chances of success. However, it has been shown that recurrence or partial failure of the operation involves mainly the intersphincteric plane (residual sepsis, secondary tracts etc) [10]. In the case that is presented here, the LIFT technique was used in order to disconnect the internal opening from the fistula tract while at the same time exploring the intersphincteric space for residual septic foci. Then, the remaining part of the fistulous tract (that is from the external sphincter muscle towards the external fistula opening) was treated with the FiLAC procedure. In this way, there was no need to core-out the remaining part of the tract (as in LIFT plus)[4], since the laser application was able to seal the fistula without any external wounding. This allowed for a faster recovery with minimal post-operative pain and the patient being able to resume normal activity in less than a week's time.

Since its first description by Wilhelm et al., back in 2011 [9], FiLAC has emerged as a minimally invasive method for treating anal fistulas, as well as pilonidal disease $[11,12]$. The circumferentially emitted laser energy destroys the fistula epithelium and simultaneously obliterates the remaining fistula tract. Giamundo et al., use the laser alone to seal the fistula tract, since its shrinkage effect is adequate to close the internal opening as well [13]. On the other hand, Wilhelm et al., add some form of manipulation regarding the internal opening $[6,9]$. In this study, FiLAC was combined with LIFT for the first time in an effort to achieve definite results, since the patient received immunomodulators and the chance of failure was believed to be greater than other individuals with the same type of fistula.

In conclusion, this is the first report of a combination therapy for transsphincteric anal fistula, applying both the FiLAC and LIFT techniques. Based on the advantages of each individual method, optimal results can be achieved, even in patients with additional risk factors for failure of primary healing or recurrence.

\section{Conflict of interest}

No authors have a conflict of interest or any financial tie to disclose.

\section{References}

1. Dudukgian $\mathrm{H}$, Abcarian $\mathrm{H}$ (2011) Why do we have so much trouble treating anal fistula? World J Gastroenterol 17: 3292-3296.

2. Narang SK, Keogh K, Alam NN, Pathak S, Daniels IR, et al. (2017) A systematic review of new treatments for cryptoglandular fistula in ano. Surgeon 15: 30-39.

3. Rojanasakul A, Pattanaarun J, Sahakitrungruang C, Tantiphlachiva K (2007) Total anal sphincter saving technique for fistula-in-ano; the ligation of intersphincteric fistula tract. J Med Assoc Thai 90: 581-586.

4. Sirikurnpiboon S, Awapittaya B, Jivapaisarnpong P (2013) Ligation of intersphincteric fistula tract and its modification: Results from treatment of complex fistula. World J Gastrointest Surg 5: 123-128.

5. Giamundo P, Esercizio L, Geraci M, Tibaldi L, Valente M, et al. (2015) Fistula-tract Laser Closure (FiLaC): long-term results and new operative strategies. Tech Coloproctol 19: 449-453.

6. Wilhelm A, Fiebig A, Krawczak M (2017) Five years of experience with the FiLaC laser for fistula-in-ano management: long-term follow-up from a single institution. Tech Coloproctol 21: 269-276.

7. Amato A, Bottini C, De Nardi P, Giamundo P, Lauretta A, et al. (2015) Evaluation and management of perianal abscess and anal fistula: consensus statement developed by the Italian Society of Colorectal Surgery (SICCR). Tech Coloproctol 19: 595-606.

8. Chand M, Tozer P, Cohen RC, (2017) Is FiLaC the answer for more complex perianal fistula? Tech Coloproctol 21: 253-255.

9. Wilhelm A (2011) A new technique for sphincter-preserving anal fistula repair using a novel radial emitting laser probe. Tech Coloproctol 15: 445449 .

10. Tan KK, Tan IJ, Lim FS, Koh DC, Tsang CB, et al. (2011) The anatomy of failures following the ligation of intersphincteric tract technique for anal fistula: a review of 93 patients over 4 years. Dis Colon Rectum 54: 13681372.

11. Dessily M, Charara F, Ralea S, Allé JL, (2017) Pilonidal sinus destruction with a radial laser probe: technique and first Belgian experience. Acta Chir Belg 117: 164-168.

12. Georgiou GK (2016) Outpatient Treatment of Pilonidal Disease with a 1470nm Diode Laser; Initial Experience. Int J Surg Surgical Proced.

13. Giamundo P, Geraci M, Tibaldi L, Valente M (2014) Closure of fistula-inano with laser--FiLaC: an effective novel sphincter-saving procedure for complex disease. Colorectal Dis 16: 110-115. 